\title{
Evaluation of the LLNL Spectrometer for Possible use with the NSTec Optical Streak Camera as a Light Gas Gun Diagnostic
}

\author{
Jonathan O'Connor and Jerald Cradick \\ oconnoji@nv.doe.gov, 1.925.960.2539 \\ September 27, 2012 \\ NSTec/Livermore Operations/Electro-Optics Systems Engineering (AA24)
}

\section{INTRODUCTION AND SUMMARY}

In fiscal year 2012, it was desired to combine a visible spectrometer with a streak camera to form a diagnostic system for recording time-resolved spectra generated in light gas gun experiments. Acquiring a new spectrometer was an option, but it was possible to borrow an existing unit for a period of months, which would be sufficient to evaluate both "off-line" and in-gas gun shots. If it proved adequate for this application, it could be duplicated (with possible modifications); if not, such testing would help determine needed specifications for another model.

This report describes the evaluation of the spectrometer (separately and combined with the NSTec LO streak camera) for this purpose. Spectral and temporal resolutions were of primary interest. The first was measured with a monochromatic laser input. The second was ascertained by the combination of the spectrometer's spatial resolution in the time-dispersive direction and the streak camera's intrinsic temporal resolution. System responsivity was also important, and this was investigated by measuring the response of the spectrometer/camera system to black body input - the gas gun experiments are expected to be similar to a $3000 \mathrm{~K}$ black body — as well as measuring the throughput of the spectrometer separately over a range of visible light provided by a monochromator. The flat field (in wavelength) was also measured and the final part of the evaluation was actual fielding on two gas gun shots.

No firm specifications for spectral or temporal resolution were defined precisely, but these were desired to be in the 1-2 nm and 1-2 ns ranges, respectively, if possible. As seen below, these values were met or nearly met, depending on wavelength. Other performance parameters were also not given (threshold requirements) but the evaluations performed with laser, black body, and successful gas gun shots taken in aggregate indicate that the spectrometer is adequate for this purpose. Even still, some (relatively minor) opportunities for improvement were noticed and these were documented for incorporation into any near-duplicate spectrometer that might be fabricated in the future.

\section{DESCRIPTION}

The spectrometer under consideration was originally designed and fabricated by EG\&G and is now in the custodianship of Lawrence Livermore National Laboratory (LLNL) High Explosive Application Facility (HEAF). If it performs as required for this task, it may be duplicated, with or without minor modifications. If not, it would serve as a baseline for either designing and fabricating or commercially acquiring a unit with higher performance. 
The spectrometer is essentially of Czerny-Turner configuration, except that in place of the usual reflecting lenses it uses two Nikon refracting lenses. Figure 1 shows the spectrometer coupled to a streak camera and a Spectral Instruments SI- 800 CCD Camera as the readout. Figure 2 is the mechanical design of the spectrometer. Collimating (input) and focusing (output) lenses are both Nikon $105 \mathrm{~mm}, \mathrm{f:1.8}$. Between the two Nikon lens is a rotation stage with a mount that can hold two reflecting diffraction gratings, $67 \mathrm{~mm}$ square, back to back. The mount is equipped with a 180 degree "flipping" mechanism, which can bring either of the defraction gratings into the beam path at will. The rotation stage (the axis of which passes through the surface of the grating being used) allows adjustment of the center wavelength. Gratings of 600 and 1200 cycles $/ \mathrm{mm}$ are currently installed (back-to-back) on the rotation stage, and all of the tests reported upon here were done with the $600 \mathrm{cycles} / \mathrm{mm}$ grating. Light enters the spectrometer via a fiber optic, which also serves as the input slit. An SMA fiber mount upstream of the collimating lens fixes the fiber-end position relative to the lens at one focal length upstream. The lens does collimate as long as it is focused at infinity, at which it is staked for assurance.

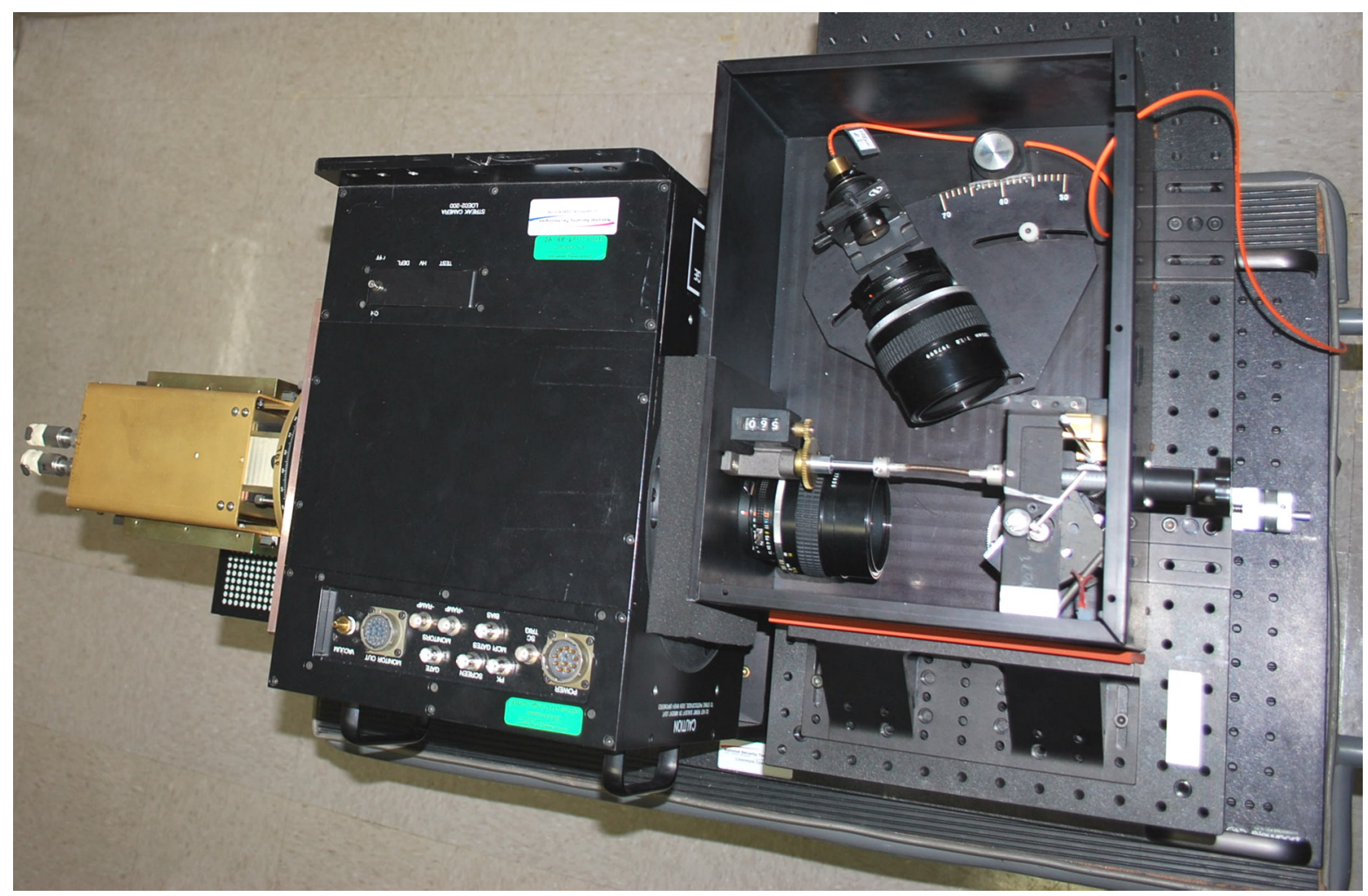

Figure 1: Streaked spectrometer coupled to streak camera and CCD. Input optical fiber (orange) enters from upper right; collimating lens is near top, grating is near lower right, and focusing lens is at lower left of spectrometer. The knob on right rotates the grating to adjust the center wavelength, which is read out by mechanical odometer located above collimating lens.

The streak camera is an NSTec/LO unit, Model LOE97-100. It is in the so-called "slow" category of this type of camera, with maximum sweep time of $30 \mu$ s and minimum of $2 \mu \mathrm{s}$. Since the expected duration of the experiment is $\sim 1 \mu$ s, the camera would be used with a $2 \mu$ s sweep (and this is where it was tested). The streak camera output image is recorded with a Spectral 
Instruments SI-800 CCD camera with a 4k x 4k array of $9 \mu \mathrm{m}$ pixels. The two cameras (streak and CCD) are coupled by a fiber optic faceplate. The spectrometer output is coupled into the streak camera by adjusting the gap between the two units so that the spectrometer output is focused on the streak camera fiber-optic faceplate -- directly, with no optics between the spectrometer and the streak camera. The NSTec LO streak cameras have a $50 \mathrm{~mm}$ active area for the streak tube. This streak camera is using a $40 \mathrm{~mm}$ micro-channel plate image (MCPI) intensifier tube which is located between the anode of the streak tube and the CCD Camera. The Spectral Instruments SI- 800 CCD Camera is a $4 \mathrm{k}$ x $4 \mathrm{k}$ with a $9 \mu \mathrm{m}$ pixel size, which equates to a $36 \mathrm{~mm}$ square; therefore, the CCD Camera records approximately 90 percent of the streaked image.

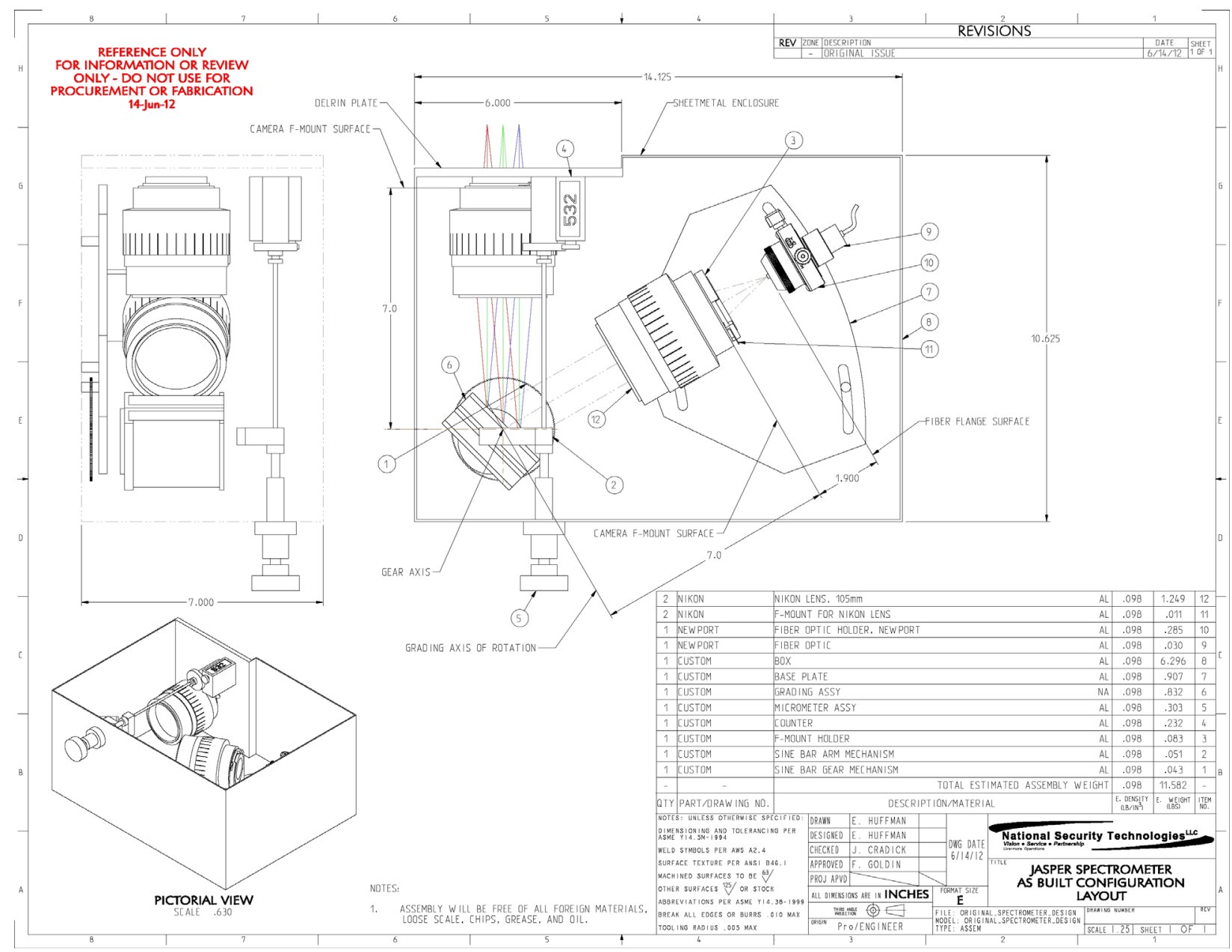

Figure 2: Mechanical design of the existing EG\&G spectrometer.

\section{PERFORMANCE}

The three major performance parameters of the spectrometer-or the spectrometer/streak camera diagnostic system - are spectral resolution, time resolution and responsivity (signal-to-noise ratio [SNR]). It is important to note that these are generally not independent of each other and can be 
traded against each other to some degree. For example, a larger input fiber core diameter will increase responsivity but decrease resolution (generally). Tests were performed using representative cases, but how performance parameters could be traded off should be straightforward. Also record length is an important parameter, but as discussed, this is fixed at $2 \mu \mathrm{s}$ across $40 \mathrm{~mm}$ (record length) given the intended application.

\section{Spectral resolution}

The streak camera component of the envisioned diagnostic is a given, but the spectrometer is optional (which is why this is an evaluation of the spectrometer) and the spectrometer spectral resolution tests were performed without the streak camera. Its output was recorded with the same SI-800 CCD camera which is normally used to record the streak camera output. This was done at two wavelengths, $693 \mathrm{~nm}$ and $543 \mathrm{~nm}$, using red and green HeNe lasers respectively. In both cases the laser output was received directly by the spectrometer input fiber (without using lens coupling). Optimal focus was found by adjusting the spectrometer/CCD camera gap and minimizing the spot size image. The full width half $\max (\mathrm{FWHM})$ of a lineout of this is taken as the measure of spectral resolution.

Table 1 below summarizes the resolution results with the data that was taken with a $50 \mu \mathrm{m}$ core fiber. This is used to back out the FWHM contribution from just the spectrometer by assuming the fiber is a flattop circle, which itself would have a FWHM of $(\sqrt{ } 3)$ (radius). (Contributions are assumed to add in quadrature). The first column is wavelength $(\lambda)$ and all others are FWHM, and all values are in nm. The "Measured" and "Streak Camera Contribution" columns are measured while the "Fiber Contribution," "Spectrometer Contribution," and "Total Fiber" columns are inferred.

\begin{tabular}{|c|c|c|c|c|c|c|}
\hline$\lambda$ & Measured & $\begin{array}{c}\text { Fiber } \\
\mathbf{( 5 0} \boldsymbol{\mu m}) \\
\text { Contribution }\end{array}$ & $\begin{array}{c}\text { Spectrometer } \\
\text { Contribution }\end{array}$ & $\begin{array}{c}\text { Streak } \\
\text { Camera } \\
\text { Contribution }\end{array}$ & $\begin{array}{c}\text { Total } \\
\text { (with 50 } \\
\boldsymbol{\mu m} \text { ) Fiber }\end{array}$ & $\begin{array}{c}\text { Total } \\
\mathbf{( w i t h} \\
\mathbf{2 0 0} \boldsymbol{\mu m}) \\
\text { Fiber }\end{array}$ \\
\hline 543 & 0.9 & 0.54 & 0.72 & 1.1 & 1.4 & 1.7 \\
\hline 693 & 1.2 & 0.53 & 1.08 & 1.1 & 1.5 & 1.8 \\
\hline
\end{tabular}

Table 1. Spectral resolution, as defined by point spread function (FWHM). All values are in nm.

Black body data taken with bandpass filters indicated dispersion in terms of $\mu \mathrm{m}$ at the CCD vs. $\mathrm{nm}$ of wavelength varied approximately $20 \%$ from $400 \mathrm{~nm}$ to $600 \mathrm{~nm}$. It is about $10 \mu \mathrm{m}(\mathrm{CCD}) /$ $\mathrm{nm}(\lambda)$ for the two wavelengths tested and this is used in finding the FWHM. A more comprehensive and accurate determination of dispersion will be done in the future with a Livermore Operations Melles Griot 43 Series Ion Laser capable of ten different visible wavelengths.

For the data that was taken with the $50 \mu \mathrm{m}$ fiber; contributions to the FWHM fiber and spectrometer are comparable. Also, tests were done with a $200 \mu \mathrm{m}$ fiber and of course the resolution drops which can be seen in Table 1. The time resolution begins to be more of a function of the fiber than the spectrometer. A larger fiber than $200 \mu \mathrm{m}$ might be considered for 
experiments if the light levels were particularly low but by this point the fiber width would completely dominate the overall resolution (assuming FWHM quadrature addition) and so it was not measured for this case. From previous testing, the spatial resolution of the streak camera plus the CCD camera varies considerably for various sweeps speeds, input light levels and MCPI tube gains. However, a FWHM of $20 \mu \mathrm{m}$ is typical and Table 1 assumes a relatively conservative 30 $\mu \mathrm{m}$. In any case, this is seen to make an insignificant contribution to the total wavelength resolution.

\section{Temporal resolution}

Both the spectrometer and the streak camera contribute to the temporal resolution (or spread) of the system and they can easily be comparable. The contribution from the spectrometer arises from the width of the image of the fiber as imaged onto the camera input. As with spectral resolution; temporal resolution itself is a function (presumably quadrature addition) of the physical diameter of the input fiber core and the optical point-spread function of (the rest of) the spectrometer. Tests indicated that a $105 \mu \mathrm{m}$ fiber is a good resolution/amplitude compromise in that signal strength with mid-level gain is in the middle of the CCD camera's dynamic range. When properly focused, the spectrometer's point spread is down to 6 CCD pixels $(54 \mu \mathrm{m})$ at a wavelength of $543 \mathrm{~nm}$ or 8 CCD pixels $(72 \mu \mathrm{m})$ at a wavelength of $633 \mathrm{~nm}$ which both are less than $1 \mathrm{~mm}$. With a nominal sweep of approximately $2.0 \mu$ s across $40 \mathrm{~mm}$ (full sweep) this corresponds to $1.6 \mu \mathrm{s}$ across the CCD camera. Note the magnification of the streak tube itself is 1:1. At the same time, the temporal resolution (50\% Contrast Transfer Function [CTF]) of just the streak camera is about $2.5 \times 10^{-3} \times$ record length or about $5 \mathrm{~ns}$. Convolving these gives a net resolution of about $5 \mathrm{~ns}$ which is listed in Table 2 . The first column is wavelength $(\lambda)$ in $\mathrm{nm}$ and all others are FWHM in ns. The "Measured" and "Streak Camera Contribution" columns are measured while the "Fiber Contribution," "Spectrometer Contribution," "Total Fiber," and "Total" columns are inferred.

Note that the real limits are signal input strength, Micro-Channel Plate (MCP) noise vs. gain, and CCD Camera responsivity, which determines the minimal fiber diameter and, therefore, streak camera resolution. Since little temporal spread is directly attributable to the spectrometer itself, there is little to be gained in this regard with any upgrades to the spectrometer.

\begin{tabular}{|c|c|c|c|c|c|c|}
\hline $\boldsymbol{\lambda}$ & $\begin{array}{c}\text { FWHM } \\
(\mathbf{n s}) \\
\text { Measured }\end{array}$ & $\begin{array}{c}\text { Fiber } \\
\mathbf{( 5 0} \boldsymbol{\mu m}) \\
\text { Contribution }\end{array}$ & $\begin{array}{c}\text { Spectrometer } \\
\text { Contribution }\end{array}$ & $\begin{array}{c}\text { Streak } \\
\text { Camera } \\
\text { Contribution }\end{array}$ & $\begin{array}{c}\text { Total } \\
\mathbf{( 5 0} \boldsymbol{\mu m} \\
\text { Fiber) }\end{array}$ & $\begin{array}{c}\text { Total } \\
\mathbf{( 1 0 5} \boldsymbol{\mu m})\end{array}$ \\
\hline 543 & 3.2 & 1.9 & 2.5 & 4.0 & 5.1 & 5.8 \\
\hline 693 & 2.4 & 1.9 & 1.4 & 4.0 & 4.6 & 6.2 \\
\hline
\end{tabular}

Table 2. Temporal Resolution. Wavelength $(\lambda)$ is in $n m$ and all other values (FWHM) are in ns.

\section{Responsivity}

Since the gas gun application is expected to be similar to a black body (at 3000K), the system's responsivity was measured using a Micron Model M390 Black Body source located at LLNL. The nominal assumption of the angular size of the application source is that it fills a Numerical Aperture (NA) of 0.22 . The ideal test is to use a collecting fiber of this aperture and overfill it 
with the black body or equivalently underfill a faster fiber with a source with a solid angle corresponding to a NA of 0.22 . However, it was impossible to bring the end of the fiber close enough to the black body source to fill this large of a NA. Therefore, the data was compensated by ratio of NA 0.22 to the effective (much less) NA of the test set-up.

Determining this $\mathrm{NA}_{\text {eff }}$ was complicated by a 1" diameter x 6" long stainless steel tube projecting from the black body oven. The black body Lambertian surface is at some point along the tube, but needed to be determined. This was done using an optical power meter (Newport Model 1918-C) to measure the radiant intensity at various distances, temperatures and wavelengths (using notch filters). These measurements lead to effective positions of the black body surface giving effective NA's for the input to the fiber. The net uncertainties from this train of measurements and analyses prevented the quantitative results from being useful for calibration but did demonstrate that the spectrometer/streak camera combination had sufficient responsivity, even at temperatures to record black body spectra in streaked mode (even well below the benchmark $3000 \mathrm{~K}$ level). The details of this method and the results obtained are presented in the document "Characterizing LLNL Black Body for use in testing streaked spectrometer" by F. Goldin.

\section{FLAT FIELD AND VIGNETTING}

This spectrometer provides for adjusting the center wavelength by changing both the grating angle and the included angle between the axes of the two lenses. However, for a short-pulse input, a single central wavelength must be selected and of course data at all wavelengths will be taken at this setting. It would be expected that in this case responsivity (throughput) vs. wavelength may be significantly peaked at the selected wavelength falling sharply to either side.

\section{Calculation}

Before considering any such responsivity measurements, a calculation may at least predict vignetting with respect to wavelength based (solely) on the optics of the spectrometer. This may be expected to dominate over streak camera/CCD camera contributions (to large-scale vignetting, not pixel-to-pixel responsivity) and would in any case provide a lower bound to the vignetting. This was done assuming the fiber input is faster (higher NA) than the collimating and focusing lenses (and the fact that the lenses are of the same speed. In this case, the collimated light from the first lens will exactly fill the second at the central wavelength and falls off as the wavelength varies due to the light footprint into the focusing lens shifting away from alignment. Thus the geometric vignetting is taken as simply given by the overlapping areas of the footprint and aperture circles.

With a central wavelength of $535 \mathrm{~nm}$ and the wavelength band recorded limited by a $38 \mathrm{~mm}$ CCD, the recorded band will be from 283 to $783 \mathrm{~nm}$. In this case, the overlap area will of course be 1.00 at $535 \mathrm{~nm}$ and fall to 0.77 at $283 \mathrm{~nm}$ and $0.78 \mathrm{~nm}$ at $783 \mathrm{~nm}$. As discussed below, one modification to the system would be to add optical comb inputs on either side of the streak camera input slit for a time scale. Assuming this to take up approximately $2 \mathrm{~mm}$ on either edge of the recorded image, the full band would then be about 308 to $758 \mathrm{~nm}$ with about 0.80 throughput at both these extremes. 


\section{Measurement}

It was initially intended to measure this curve with the black body source, but by the time an optical train was in place to limit the input fiber's field of view to the source interior, the black body source failed at LLNL. An alternate flat field source was a Melles Griot 43 Series Ion Laser which provides ten visible wavelengths. Its power and monochromaticity would be very advantageous but the system was not available in time for this evaluation although it should be used for future work. The third option, which was in fact used, was a Gamma Scientific GS4100 monochromator. This can provide input from about 300 to $800 \mathrm{~nm}$ and was used for wavelength responsivity measurements. This is a continuous source, but at low power levels $(\sim 1-10 \mathrm{nW})$. The output measurements were made with a Newport Model 1918-C power meter.

Due to the low power available from the monochromator, a $400 \mu \mathrm{m}$ core fiber was used rather than the $105 \mu \mathrm{m}$ fiber used in the first fielding, cited below. The core diameter would be expected only to affect the total power coupled into the fiber from the monochromator; therefore, not affecting the transmission fraction. However, the NA of the fiber might affect the shape of the transmission curve because the fiber NA will determine if the lens (which has an NA of 0.28) is over or under-filled in acceptance angle. The result is depicted in Figure 4. It may be noted that the very significant falloffs at either end; especially at the short end, might be slightly exaggerated due to the fact that the extremely low power levels made alignment more difficult at the ends then in the central region. (The beam could not be visually seen and alignment of the spectrometer output beam to the detector was done by maximizing the power reading vs. position). The curve certainly still does represent an upper bound to the non-flatness of the spectrometer.

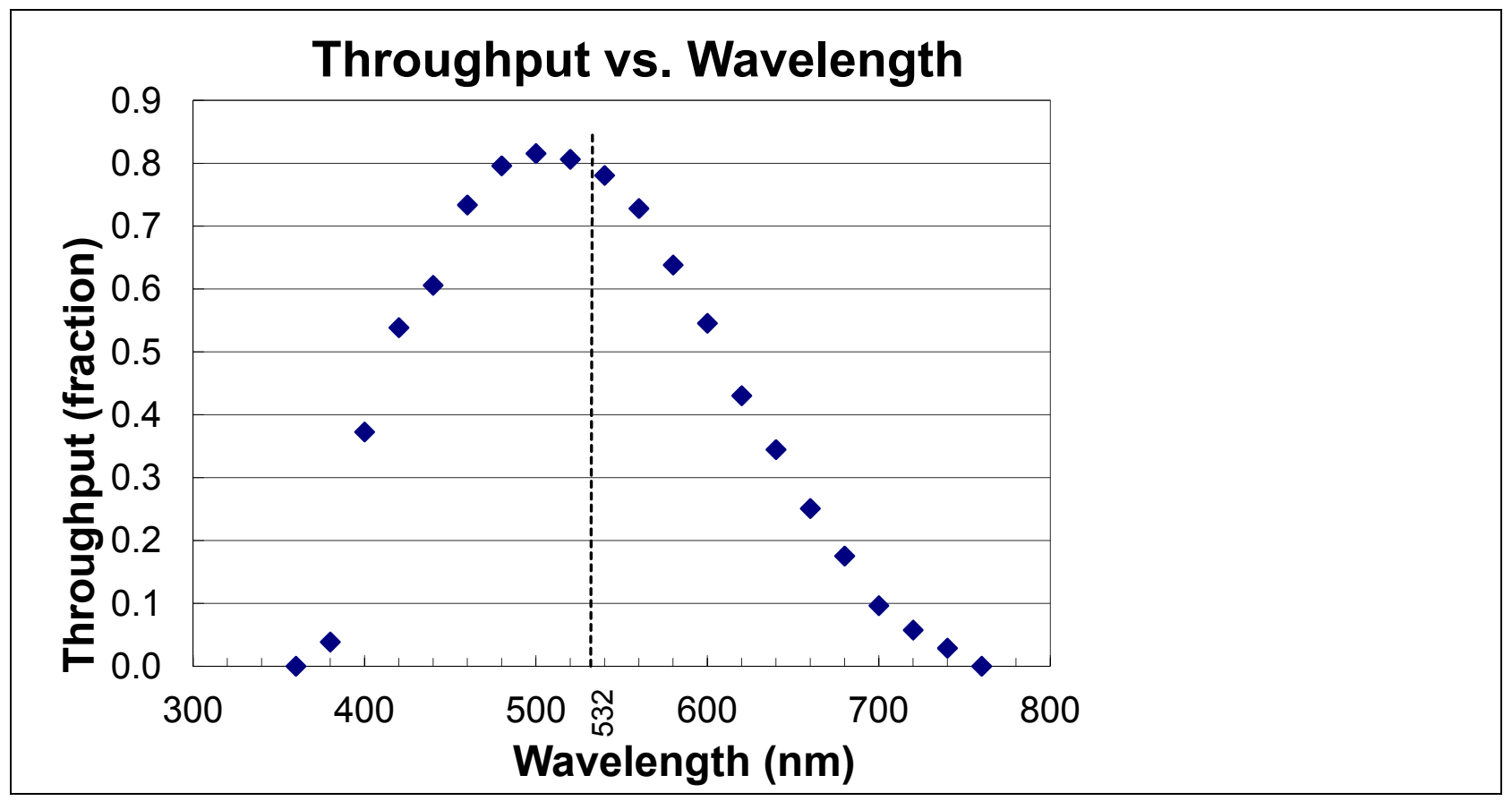

Figure 3: Wavelength responsivity as measured with monochromator input. Underfilled power meter measures spectrometer output normalized by power input from fiber. Angles for the collimating lens and grating are set for a central wavelength of $532 \mathrm{~nm}$. 


\section{FIELDING ON GAS GUN AT LLNL HEAF}

The system was tested, calibrated and then successfully fielded on two shots at the HEAF twostage light gas gun at LLNL on May 23 and 24, 2012. The target was a quartz crystal which the diagnostic viewed from the back side. The data, namely the time-resolved spectrum, showed the dynamic response of the crystal to the shock wave as it propagated through the material.

The data image from the first shot is shown in Figure 4. Wavelength increases from bottom to top, and time is left to right - the reference light dots at the top are $50 \mathrm{~ns}$ apart. The semi-bright zone at left is when the projectile first hits target, the end of the light zone near the middle of image is when the shock wave exits target and the ultra-bright zone on the right side of image is when projectile/crystal material strikes the optical fiber.

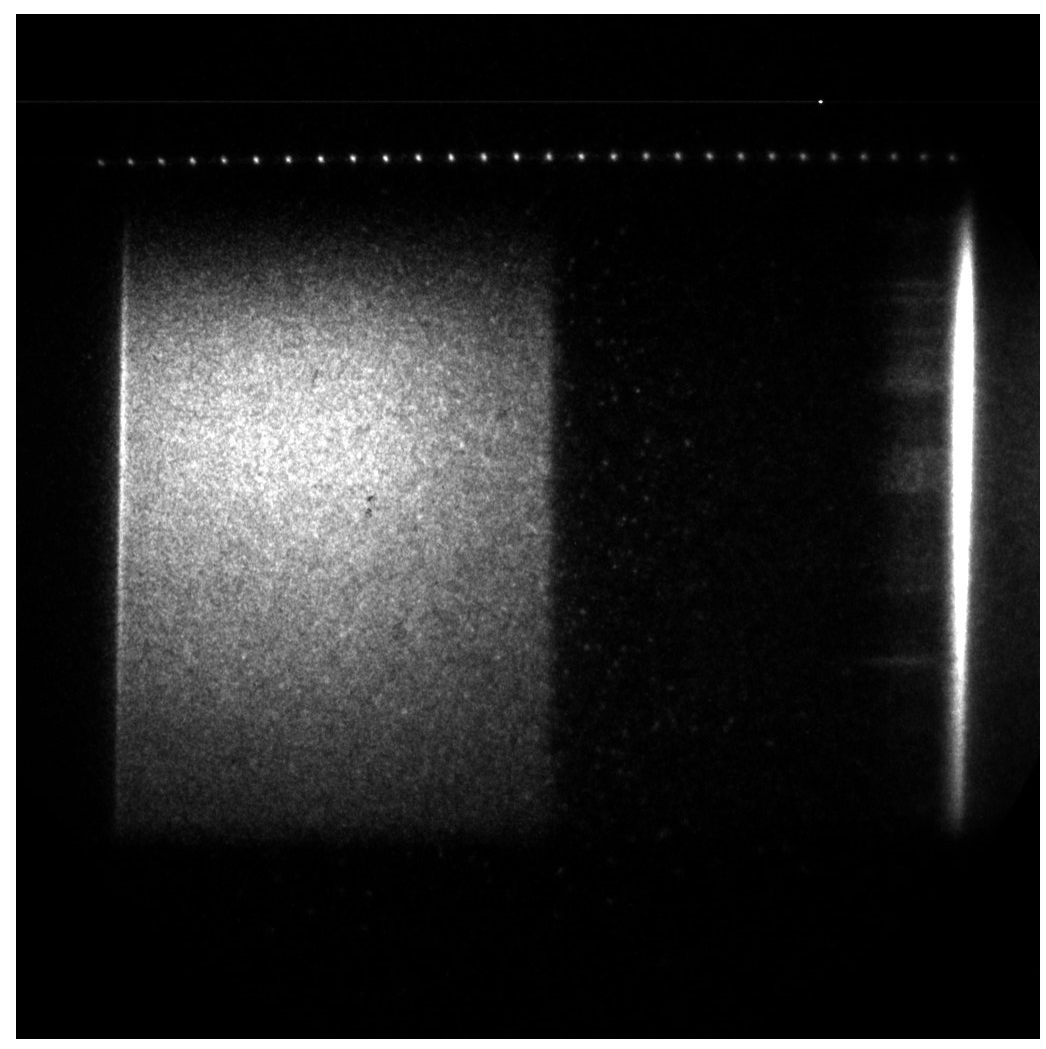

Figure 4: Streaked spectrum from first fielding of system at LLNL HEAF light gas gun shot. Wavelength increases from bottom to top and time is from left to right.

Optimizing the trade-offs of MCP gain (signal amplitude vs. noise) and fiber core diameter (signal amplitude vs. resolution) were resolved with black body testing. The parameters chosen based on this were a MCPI gain of $800 \mathrm{~V}$ (middle of normal range) and a $105 \mu \mathrm{m}$ diameter fiber. These resulted in maximum light signal levels of about half of the dynamic range of the recording CCD camera. (The camera saturates later in time, when the projectile impacts the fiber but this is not part of the experiment data.) The maximum recording limit is 32,000 counts (CCD camera saturation), so these represent the center of the effective dynamic range for good (if slightly conservative) data collection. 


\section{SPECTROMETER PHYSICAL CHARACTERISTICS AND POSSIBLE FUTURE MODIFICATIONS}

In using the system, a few minor but helpful modifications for any possible duplication of the spectrometer were noted. These are essentially mechanical in nature and include:

1. Remove the "dog leg" in the output side of the enclosure for simpler coupling to the streak camera.

2. Add holders for ND filters in the internal beam path (between the lenses).

3. Modify the grating mount to make swapping the gratings easier and additionally incorporate a kinematic mount to ensure a reproducible position.

4. Integrate fiber inputs in to the front of the streak camera for both the optical comb (both sides) and the fiducial mark (one side).

5. Simplify the drive for the grating rotation by replacing the sine-bar mechanism with a "linear" rotation stage. If the stage is to be manual (the presumption at least initially), then the relationship between the grating angle and the center wavelength would be given by an equation or a table. If the stage is to be computer controlled, then the wavelength would be displayed.

6. Make swapping input fiber more convenient by eliminating the need for moving the mount.

7. Make light-tight nonconductive seals at the spectrometer fiber input and between the spectrometer and the streak camera.

8. Add extensions to the bottom surface with means (probably slotted holes) for affixing the spectrometer to the optical breadboard.

\section{CONCLUSION}

The general conclusion is that this spectrometer with the output recorded by the streak camera/CCD camera has sufficient performance for useful time-resolved spectral measurements on at least the subset of light-gas gun experiments intended for it. In amplitude, this is verified by being in the heart of the CCD camera recording range in actual shots and spectral and temporal resolutions tested off-line were to be about in the initially desired ranges. Also, the grating may be easily swapped for other units of the same size $(67 \mathrm{~mm})$ to vary the bandpass width and using Nikon refracting lenses allows easy swapping to vary the focal length and/or speed. In conclusion, duplicating the design with minor modifications such as those outlined above is a viable option and meets the LLNL customer's specifications for use on future JASPER experiments.

\section{DISCLAIMER}

This report was prepared as an account of work sponsored by an agency of the U.S. Government. Neither the U.S. Government nor any agency thereof, nor any of their employees, nor any of their contractors, subcontractors or their employees, makes any warranty or representation, express or implied, or assumes any legal liability or responsibility for the accuracy, completeness, or usefulness of any information, apparatus, product, or process disclosed, or 
represents that its use would not infringe privately own rights. Reference herein to any specific commercial product, process, or service by trade name, trademark, manufacturer, or otherwise, does not necessarily constitute or imply its endorsement, recommendation, or favoring by the U.S. Government or any agency thereof. The views and opinions of authors expressed herein do not necessarily state or reflect those of the U.S. Government or any agency thereof. 